\title{
Self-Sterilizing Laser-Induced Graphene Bacterial Air Filter-
}

\section{Supporting Information}

${ }^{\#}$ Michael G. Stanford, ${ }^{1}{ }^{\#}$ John T. Li, ${ }^{1}$ Yuda Chen,${ }^{1}$ Emily A. McHugh, ${ }^{1}$ Anton Liopo, ${ }^{1,5}$ Han Xiao, ${ }^{1}$ James M. Tour ${ }^{1,2,3,4 *}$

${ }^{1}$ Department of Chemistry, ${ }^{2}$ Smalley-Curl Institute and the NanoCarbon Center, ${ }^{3}$ Department of Materials Science and NanoEngineering, and ${ }^{4}$ Department of Computer Science, Rice University, 6100 Main Street, Houston, Texas 77005, United States

${ }^{5}$ Institute of Biosciences and Technology, Texas A\&M Health Science Center, Houston, Texas 77030, United States

*Corresponding Author (Email: tour@ rice.edu)

\#These authors contributed equally to this work. 


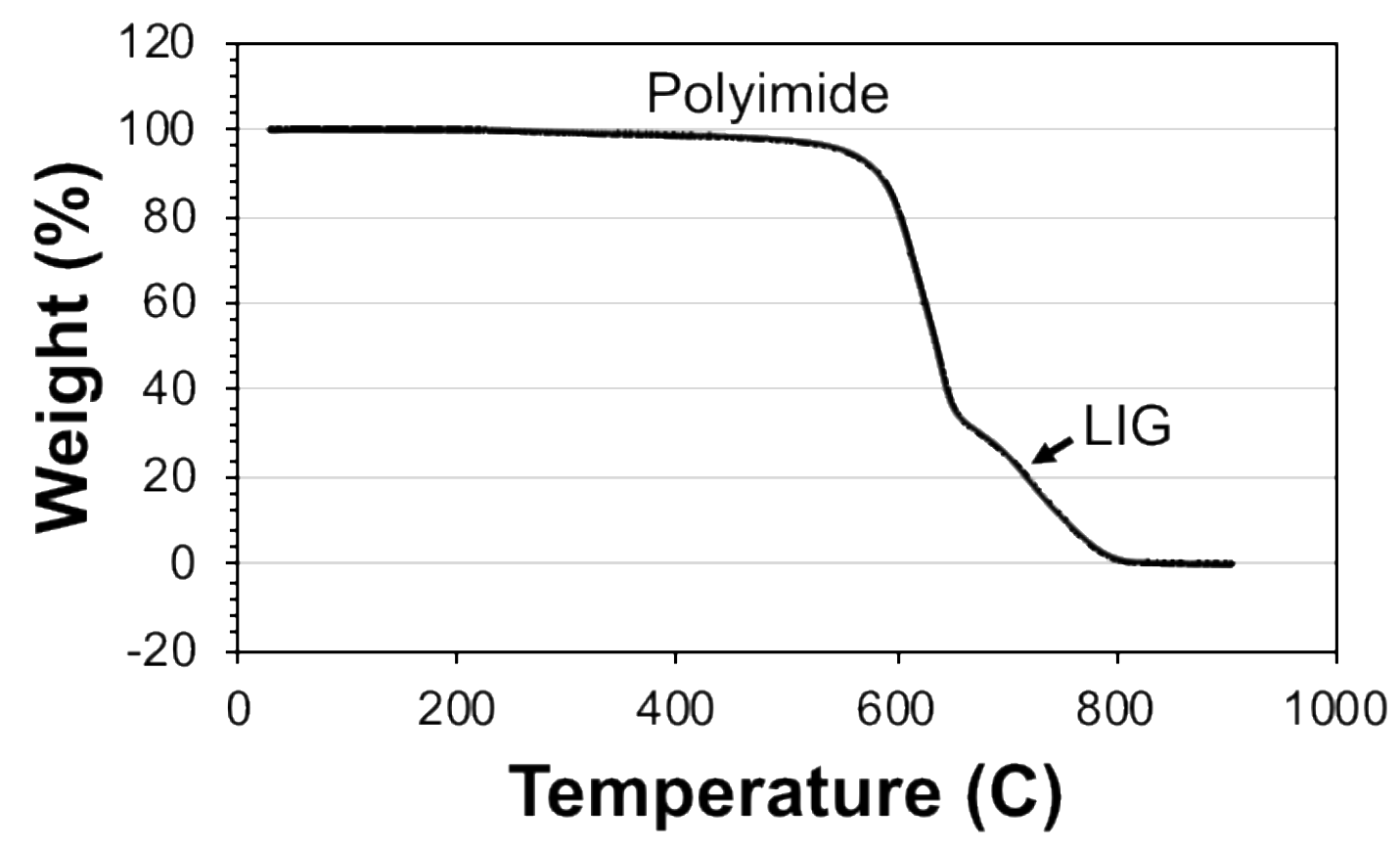

Supporting Figure S1. Analysis of the LIG filter. Thermogravimetric analysis (TGA) plot indicating that the temperature of decomposition of the PI portion of the filter is $\sim 580{ }^{\circ} \mathrm{C}$, whereas LIG begins to decompose at $\sim 660{ }^{\circ} \mathrm{C}$. PI comprises $\sim 65 \%$ of the LIG filter by mass, with the remainder being LIG. 
Attach to Sterile $0.22 \mu \mathrm{m}$ PES Vacuum Filtration Funnel

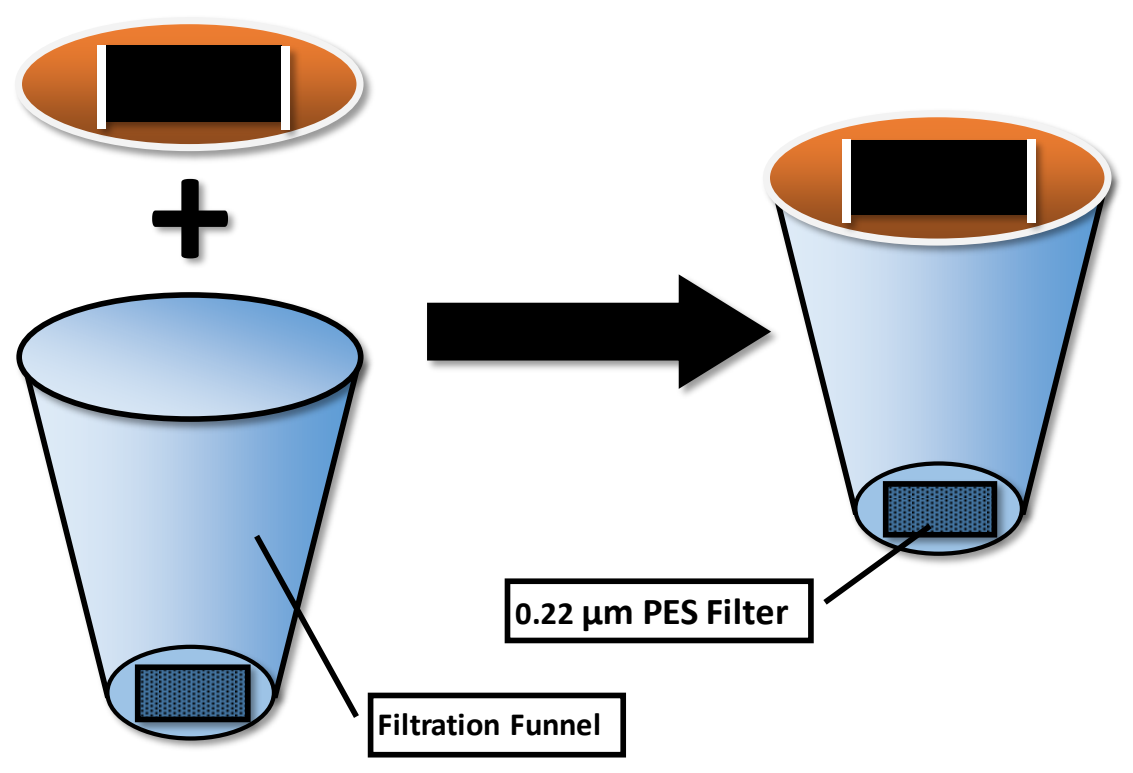

Supporting Figure S2. Schematic of the Corning 431097 vacuum filtration funnel with the LIG filter mounted over the opening. 


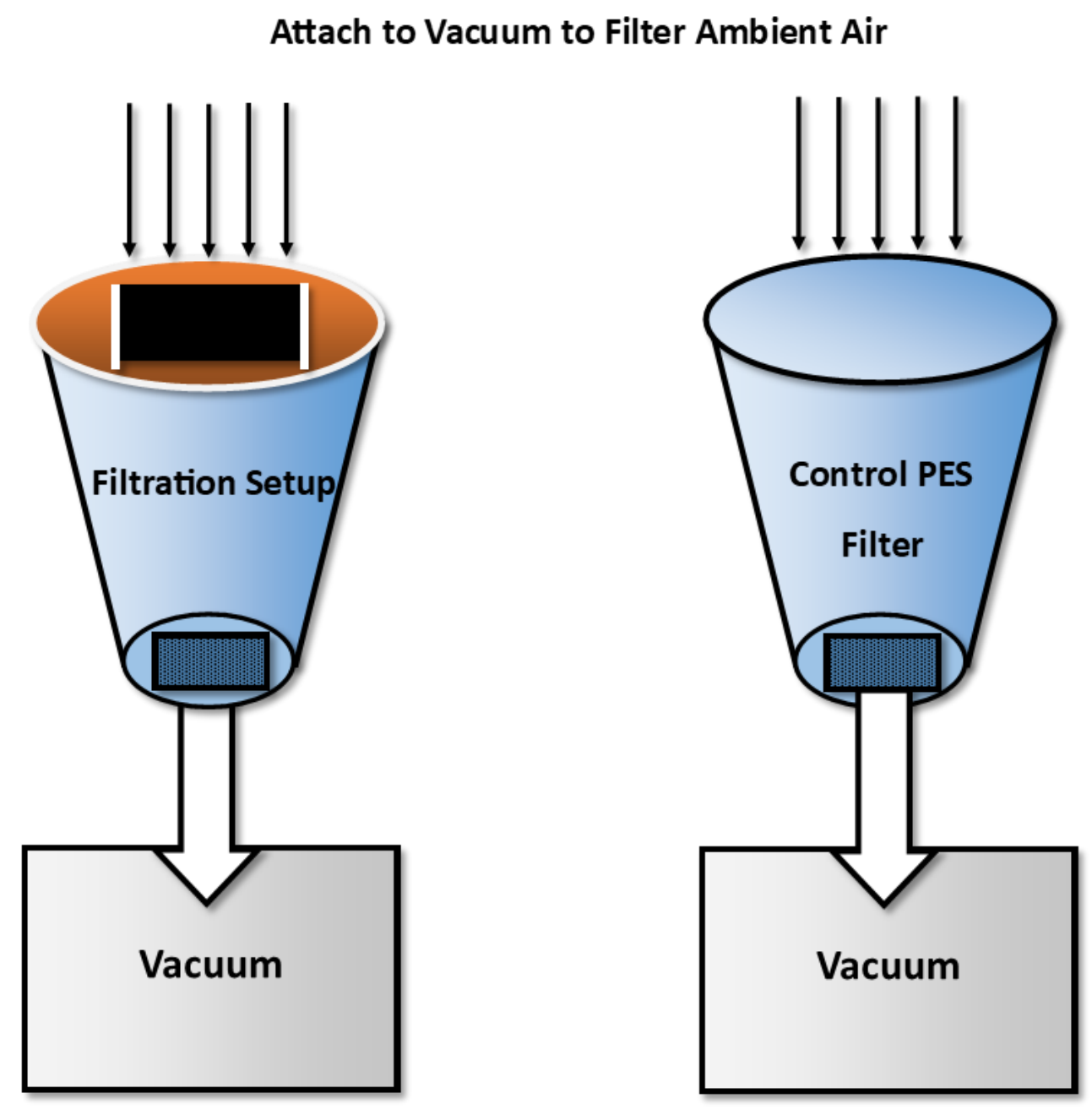

Supporting Figure S3. Schematic of airflow bacteria capture setup. Air was simultaneously pulled through the vacuum filtration funnel with the LIG filter and the control vacuum filtration funnel for $90 \mathrm{~h}$ at equal flow rate of $10 \mathrm{~L} \cdot \mathrm{min}^{-1}$. 


\section{Section Into Samples of Equal Area, Stored in Sterile Tubes}

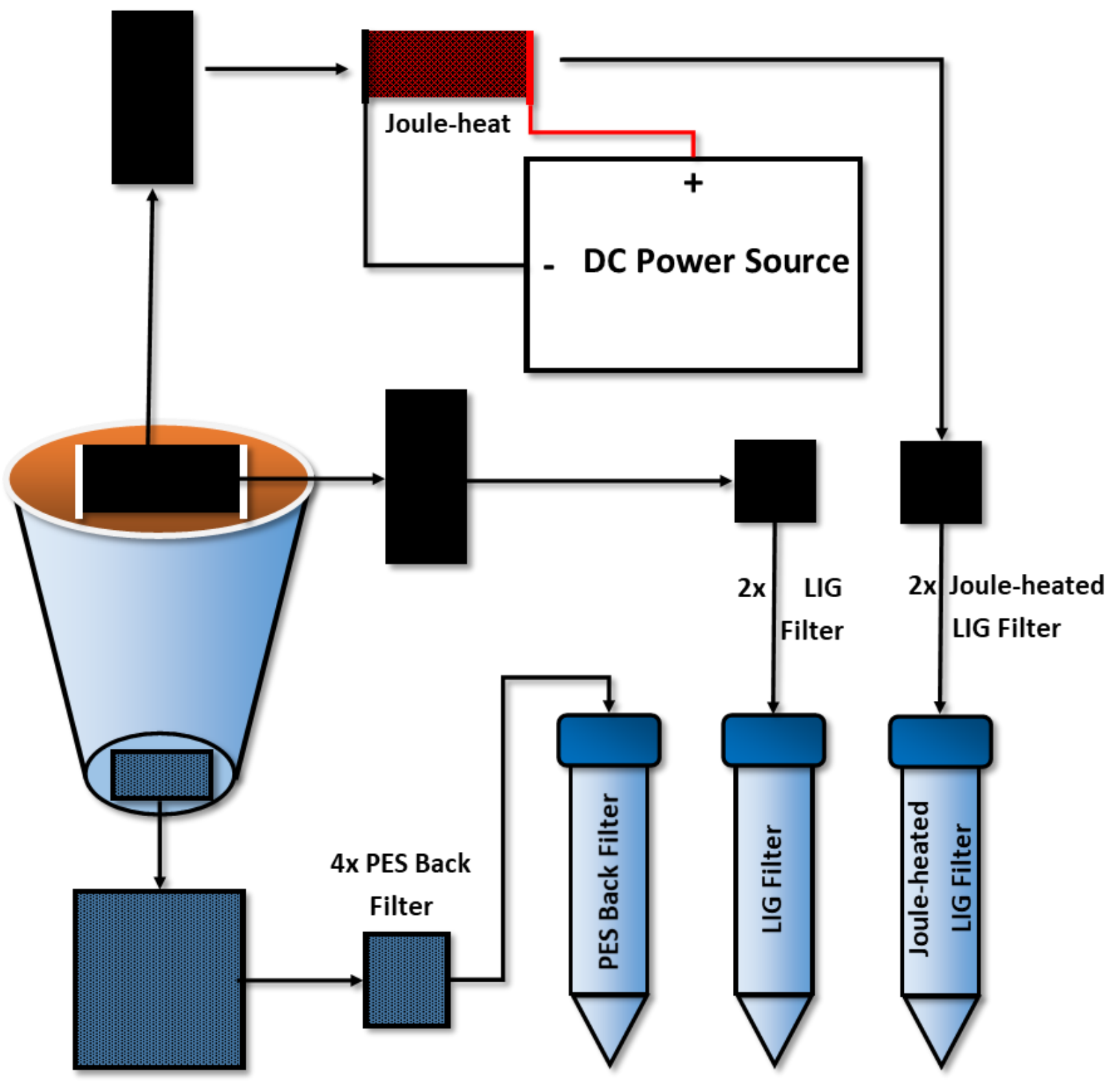

Supporting Figure S4. Schematic of the various samples that originate from the vacuum filtration funnel with the LIG filter. The control PES filter of the control vacuum funnel is sectioned analogously to the PES filter that is protected by the LIG filter. The sterile LIG control filter is sectioned into four pieces directly following photothermal conversion of the PI film into LIG. 

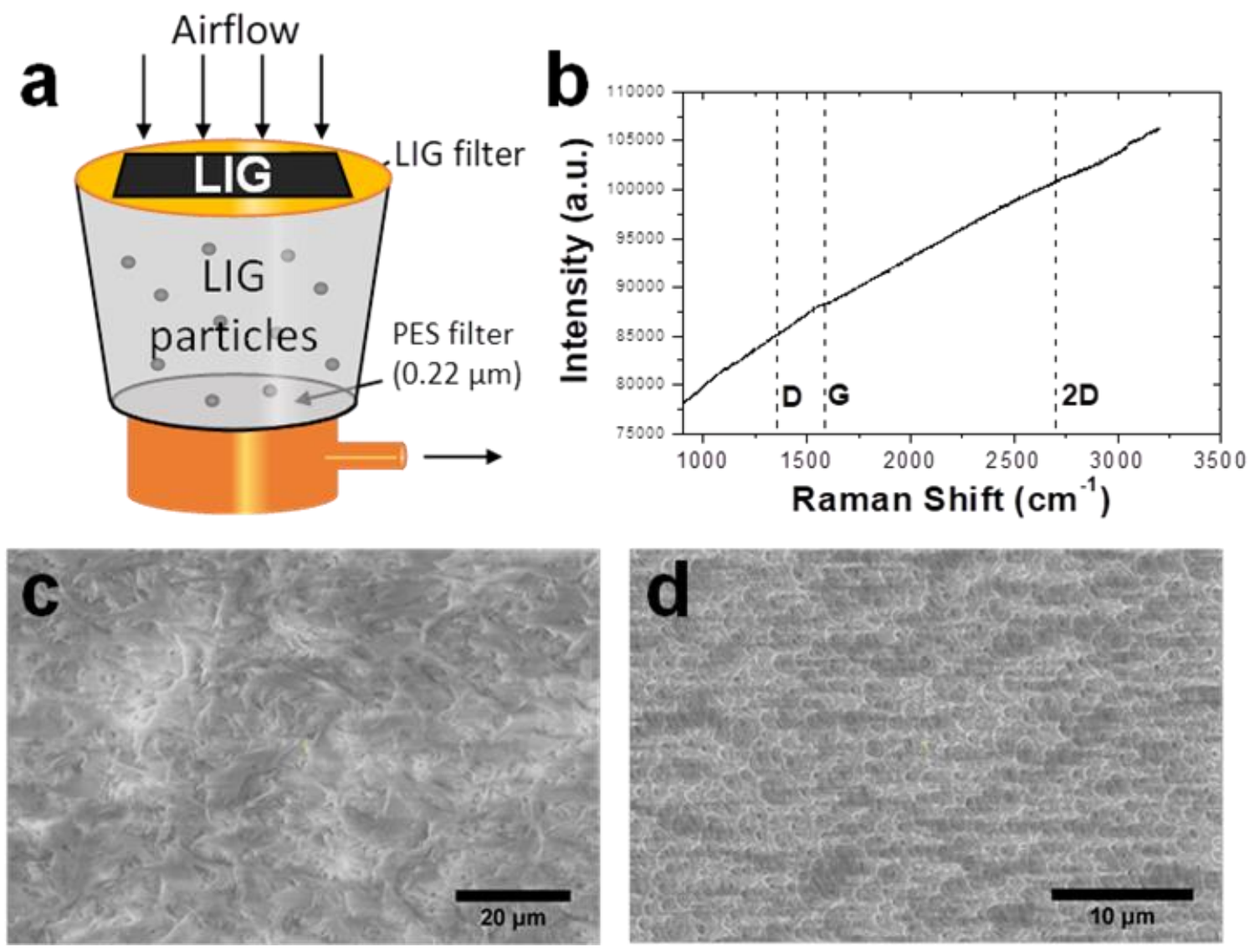

Supporting Figure S5. Expected trajectory of hypothetical LIG particles released during airflow, along with spectroscopic and microscopic evidence against the existence of such LIG particles. (a) Schematic illustrates that if LIG particles were released during airflow, the particles will be found on the PES filter membrane. Thus, as shown in (b), (c), and (d), after airflow for $90 \mathrm{~h}$, the Raman shift at $532 \mathrm{~nm}$ was measured for different portions of the PES filter membrane, and SEM images were taken. There was no 2D peak in the Raman spectrum that would indicate the presence of LIG, and no LIG particles were visible by SEM. 

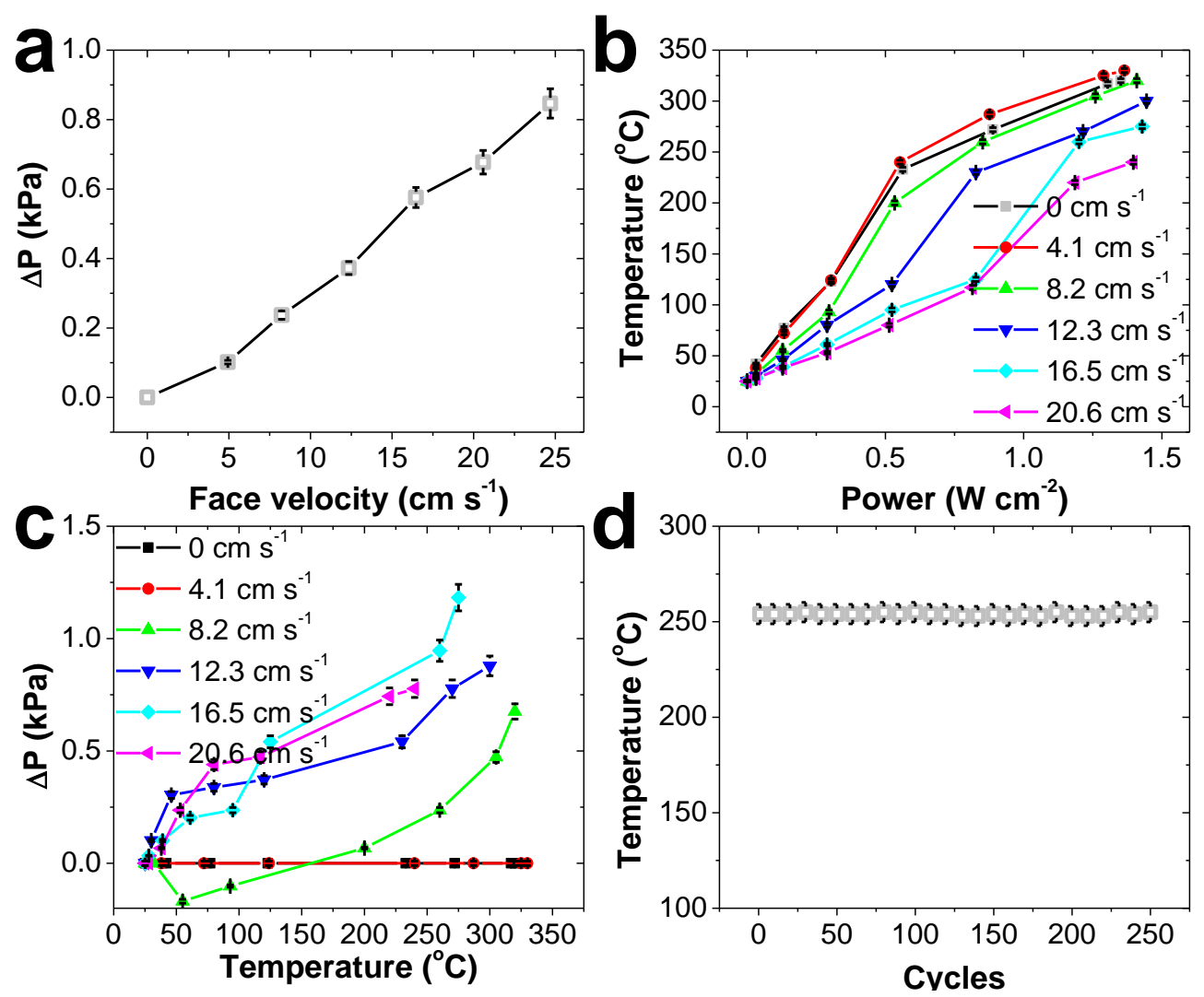

Supporting Figure S6. Temperature, power, pressure drop, flow rate, and cycling stability using a filter as shown in Figure 1c. (a) Pressure drop across the LIG filter $v$ s face velocity without Jouleheating. (b) Temperature of LIG filter vs power supplied at different air face velocities. (c) Temperature $v s$ pressure drop data for the LIG filter. (d) Joule-heating cycle stability of the LIG filter for 250 cycles at a constant potential of $15 \mathrm{~V}$ between room temperature and $255^{\circ} \mathrm{C}$, which is sufficient for biomolecule destruction. All error bars in the figure report error in measurement technique. 

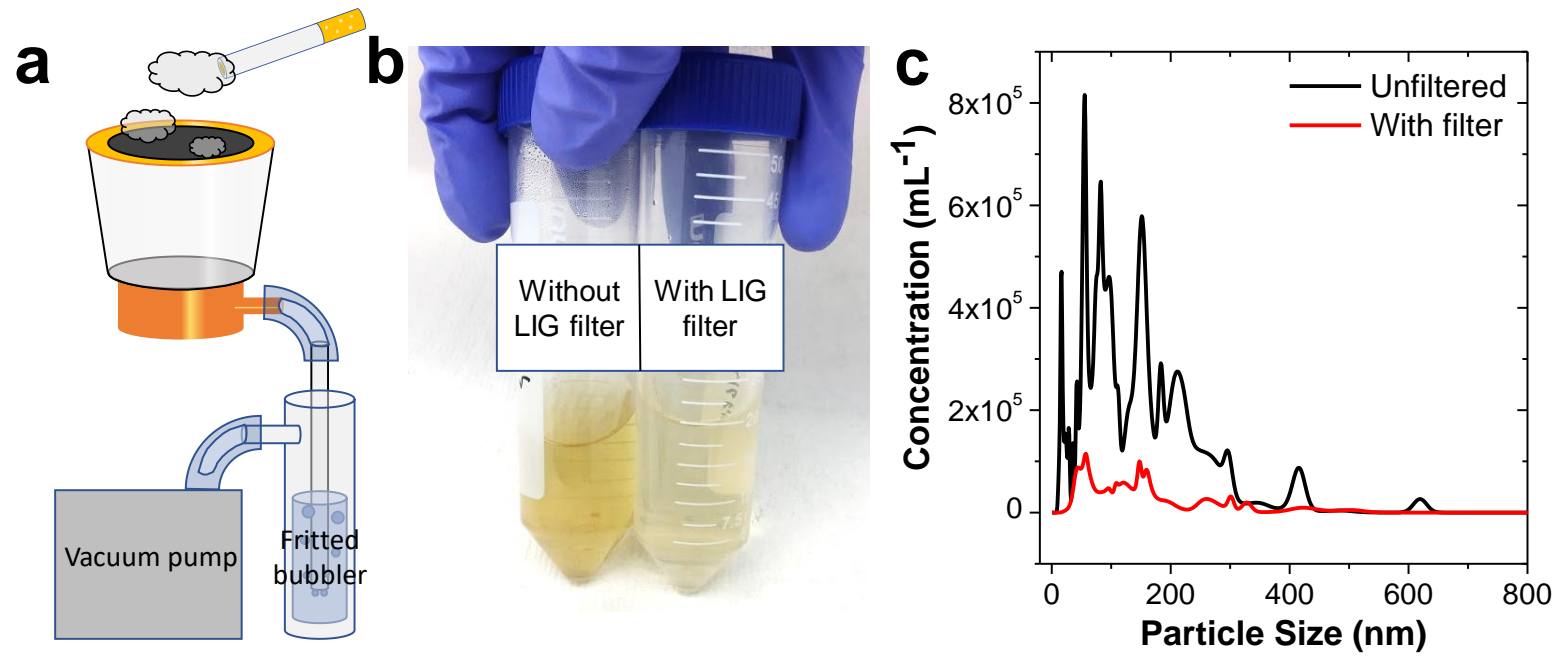

Supporting Figure S7. Particle size capture analysis. (a) Schematic of particle size capture test. (b) Optical image of the fluid inside fritted bubbler without and with the LIG filter. (c) The nanoparticle tracking analysis particle size distributions, indicating the effectiveness of the LIG filter at capturing sub-800 $\mathrm{nm}$ particles. 

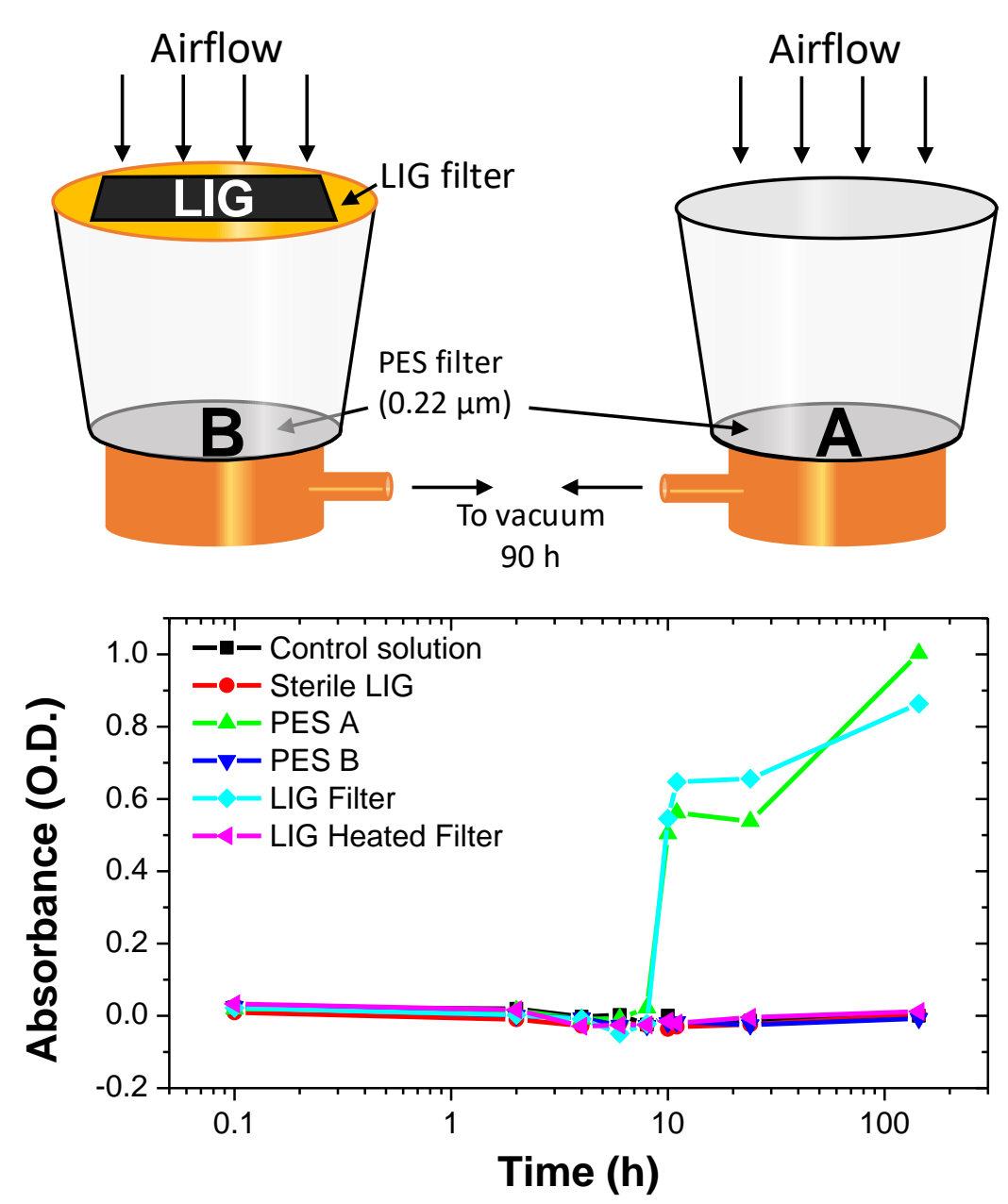

Supporting Figure S8. Airflow bacteria capture schematic and results. Schematic of the airflow bacteria capture setup, with a graph of the optical density at $600 \mathrm{~nm}$ over time of incubation in 10 $\mathrm{mL} 2$ YT medium. Samples were mildly sonicated for $2 \mathrm{~min}$ prior to incubation to free bacteria from encapsulation by LIG. Note that the proliferation of bacteria was observed for the LIG Filter and PES Filter A at 9-10 h. However, no proliferation of bacteria was observed for the Sterile LIG control filter, PES B, and the Joule-heated LIG filter. This result supports the conclusions that the LIG filter is effective at capturing bacteria, protecting PES Filter B from contamination, and Jouleheating after airflow sterilizes the LIG filter. 

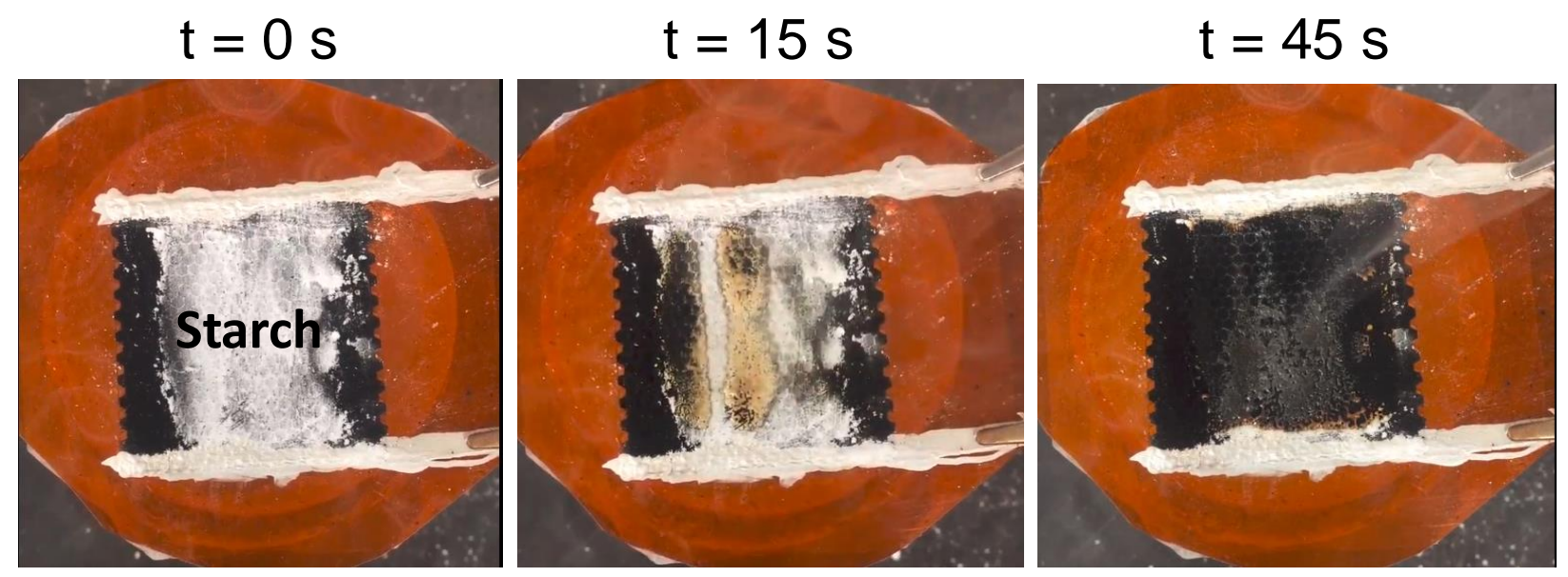

Supporting Figure S9. The carbonization of polysaccharides. Decomposition of corn starch at $300{ }^{\circ} \mathrm{C}$, demonstrating ability to carbonize polysaccharides. 